\title{
Cystatin $F$ as a key family 2 cystatin subunit and prognostic biomarker for early-stage pancreatic ductal adenocarcinoma
}

\author{
CHENGKUN YANG, TINGDONG YU, ZHENGQIAN LIU, XINPING YE, XIWEN LIAO, XIANGKUN WANG, \\ CHUANGYE HAN, GUANGZHI ZHU, WEI QIN and TAO PENG
}

\begin{abstract}
Department of Hepatobiliary Surgery, The First Affiliated Hospital of Guangxi Medical University, Nanning, Guangxi 530021, P.R. China
\end{abstract}

Received November 5, 2018; Accepted April 17, 2019

DOI: 10.3892/or.2019.7135

\begin{abstract}
Pancreatic ductal adenocarcinoma (PDAC) is one of the most lethal malignancies, and early diagnosis and assessment may enhance the quality of life and survival of patients. The prognostic value of key family 2 cystatins subunit in PDAC patients remains unknown. The potential molecular roles of family 2 cystatins and related pathways were investigated using bioinformatics analysis. The relationship of family 2 cystatin expression levels and clinical outcomes of 112 patients with early-stage PDAC were evaluated via univariate and combined survival analysis. A prognostic nomogram model was also constructed and gene set enrichment analysis was performed to investigate potential pathways in PDAC. The pathways, interaction networks, and Gene Ontology term analysis of the cystatin gene family were analyzed in the present study. Cystatin F (CST7) was identified as the key subunit of family 2 cystatins in survival analysis. PDAC patients who harbored a higher expression level of CST7 had a lower risk in overall survival (adjusted $\mathrm{HR}_{\mathrm{OS}}=0.44,95 \% \mathrm{CI}=0.25-0.77, \mathrm{P}=0.004$ ) and a longer survival time in various subgroups. The prognostic nomogram indicated that the CST7 expression model
\end{abstract}

Correspondence to: Dr Tao Peng, Department of Hepatobiliary Surgery, The First Affiliated Hospital of Guangxi Medical University, No. 6 Shuangyong Road, Nanning, Guangxi 530021, P.R. China

E-mail: pengtaogmu@163.com

Abbreviations: PDAC, pancreatic ductal adenocarcinoma; CST7, cystatin F; STRING, Search Tool for the Retrieval of Interacting Genes/Proteins; GO, Gene Ontology; DAVID, Database for Annotation, Visualization and Integrated Discovery; MERAV, Metabolic gEne RApid Visualizer; GTEx, Genotype-Tissue Expression; TCGA, The Cancer Genome Atlas; AJCC, American Joint Committee on Cancer; OS, overall survival; DFS, disease-free survival; HR, hazard ratio; CI, confidence interval; C-index, concordance index; GSEA, gene set enrichment analysis; FDR, false discovery rate

Key words: pancreatic ductal adenocarcinoma, CST7, prognosis, survival analysis, biomarker effectively predicted the outcomes of patients with early-stage PDAC (predictive ability $>0.75$ ). In the gene set enrichment analysis, it was revealed that CST7 expression may be involved in immune regulation and be associated with cell adhesion. CST7 could be a useful biomarker for the prognostic prediction of early-stage PDAC after pancreaticoduodenectomy.

\section{Introduction}

Pancreatic cancer is an aggressive disease with an extremely poor clinical outcome and a 5-year survival rate of $<5 \%(1,2)$. Approximately 37,170 new cases of pancreatic cancer were diagnosed in 2007 and nearly 33,370 mortalities were caused by this disease in the US (3). More than $80 \%$ of pancreatic ductal adenocarcinoma (PDAC) patients (accounting for $85 \%$ of pancreatic cancer) are diagnosed at a stage that is already regional or distant metastasized (4). The 5-year survival rate for PDAC, improves from 2 to $23 \%$ if the disease is diagnosed at its localized stage compared to a distant metastatic stage (5). Chemotherapy is an alternative for unresectable PDAC; however, limited survival benefit still exists. Thence, it is extremely significant to unearth novel diagnostic biomarkers for PDAC, which can indicate the use of curative surgical treatment (6).

Cysteine proteases, expressed widely in tissues, are a group of intracellular proteins with protein degradation activity which are associated with a wide variety of biological processes, including inflammation, modulation of the immune response and facilitating the progression of malignant tumors (7-9). Previous clinical studies have revealed that the cystatin superfamily proteins inhibit the proteolytic activity of cysteine proteases specifically in attenuating the aggressiveness of various malignant tumors (10-12).

Among the three distinct subfamilies belonging to the cystatin superfamily, the family 1 cystatins, represented by cystatin A (CSTA) and B (CSTB), lack disulfite bonds as well as signal peptides and only function intracellularly. Family 2 cystatin subunits are secreted proteins, all composed of 115-120 amino acids with two interchain disulfide bonds. L- and H-kininogens of family 3 cystatin subunits are complex glycosylated cytoplasmic proteins with type-2-like cystatin domains and bradykinin moiety (13-15).

There are seven members in the family 2 cystatins, cystatin SN (CST1), SA (CST2), C (CST3), S (CST4), D (CST5), 
E/M (CST6), and F (CST7). Family 2 cystatins are types of cysteine protease inhibitors found in various human fluids and secretions that appear to provide protection. To date, the expression level of the family 2 cystatins has been reported to be associated with tumor biology function, progression and prognosis in breast, small-cell lung and colorectal cancer (16-19). However, the key family 2 cystatins subunit in PDAC patients remains unknown.

In the present study, we aimed to investigate the clinical implications of the family 2 cystatins in early-stage PDAC using bioinformatics and survival analysis. Finally, CST7 was identified as a key cystatin subunit. Furthermore, a prognostic model for patients with early-stage PDAC was constructed.

\section{Materials and methods}

Biological function of the cystatin gene family. To investigate the potential molecular roles and related pathways of the cystatin gene family, we first analyzed gene-gene interactions using GeneMANIA (http://www.genemania.org/). Protein-protein interactions of cystatin genes were ascertained by the Search Tool for the Retrieval of Interacting Genes/Proteins (STRING, https://string-db.org/). Gene Ontology (GO) terms annotation and gene function enrichment analysis of cystatin genes were performed using the Database for Annotation, Visualization and Integrated Discovery (DAVID, https://david.ncifcrf. gov/home.jsp) version 6.8.

Cystatin gene family expression in tissues. Expression of CST mRNA between paraneoplastic tissues and primary cancer tissues was performed by Metabolic gEne RApid Visualizer (MERAV, http://merav.wi.mit.edu/). To investigate the cystatin gene family expression level in various normal tissues, we generated a heatmap using the Genotype-Tissue Expression (GTEx) portal (https://www.gtexportal.org/). Co-expression relationships between CST genes in tumor tissues were assessed by the Pearson correlation coefficient. Pearson correlation was performed using corrplot $\mathrm{R}$ package (available from https://github.com/taiyun/corrplot).

Cystatin gene family in survival analysis of PDAC patients. In the survival analysis, patients were divided into low- and high-level groups according to median mRNA expression level. We screened key cystatin isoforms that were associated with the prognosis of early-stage PDAC in The Cancer Genome Atlas (TCGA; https://portal.gdc.cancer.gov/). All the expression data for cystatin and the relevant clinical parameters were obtained from the University of California, Santa Cruz Xena browser (UCSC Xena: http://xena.ucsc.edu/). The inclusion criteria of patients enrolled in the present study were as follows: i) available survival data; ii) patients who underwent pancreaticoduodenectomy and the histology type was confirmed as PDAC; iii) stage I or II PDAC according to the 7th American Joint Committee on Cancer (AJCC); and iv) the tissues samples were collected by resection or biopsy prior to receiving chemotherapy. The number of AJCC stage III or IV patients was 8. To control bias, PDAC patients with AJCC stage III or IV and those non-PDAC histology types were excluded. Inclusion and exclusion criteria were implemented in accordance with a previous study (20). Finally, CST7 was selected as a key cystatin isoform in further analysis. Overall survival (OS) and disease-free survival (DFS) was calculated using SPSS version 24.0. Prognosis risk was established on CST7 expression level and trend direction depended on a regression coefficient $(\beta)$ as the positive and negative correlation that was derived from a univariate Cox proportional hazards regression model. Hazard ratios (HR) and 95\% confidence intervals (CIs) were also computing in the hazards regression model. Combined survival analysis was performed to identify the relationship between CST7 expression and clinicopathological features in the prognosis of patients with early-stage PDAC. Multivariate regression analysis of CST7 expression in PDAC patients was adjusted for prognosis-related clinical factors from univariate Cox proportional hazards regression model.

Prognostic nomogram conduction. Based on the examination and transformation of variables evaluated in a univariate Cox proportional hazards regression model, we formulated a prognostic nomogram model using the rms (21) R package. The prognostic performance of the nomogram was measured by concordance index (C-index). The $\mathrm{C}$-index was calculated to evaluate the performance of each model in the survival data and was considered a measure of predictive ability (22). Bootstraps with the resamples of PDAC patients were applied to predict survival probability and to obtain a more realistic bias-corrected estimate of the model coefficients and the C-index.

Gene set enrichment analysis (GSEA) for CST7 in PDAC patients. Setting CST gene expression levels as population phenotypes in GSEA (http://software.broadinstitute. org/gsea/index.jsp), we further analyzed gene expression omics predictions and assessed related pathways and molecular mechanisms in PDAC patients. A nominal P-value $<0.05$ and false discovery rate (FDR) $<0.25$ of the enrichment gene sets in the analysis were considered statistically significant.

Statistical analysis. All statistical analyses were performed using SPSS version 24.0 (SPSS; IBM Corp., Armonk, NY,USA) and R3.4.1 (www.r-project.org). A two-sided P-value of $<0.05$ was considered statistically significant. The survival curves and heatmap were depicted by GraphPad Prism7.01 (GraphPad Software, Inc., La Jolla, CA, USA). The Kaplan-Meier survival curves were compared by the log-rank test.

\section{Results}

The pathways, interaction networks and GO term analysis of the cystatin gene family. Using the gene-gene interaction analysis in GeneMANIA, we identified that the cystatin gene family mainly shared protein domains with other molecules, such as CSTA, CSTB and FETUB (Fig. 1A). Furthermore, protein-protein interaction networks by STRING indicated that cystatin proteins may be associated with immune proteins, such as human lymphocyte antigen (Fig. 1B). GO term enrichment analysis revealed that the cystatin family encoded products which play roles in the extracellular region and contains inhibitory activity against the peptidase and endopeptidase of the enzyme (Fig. 1C). 

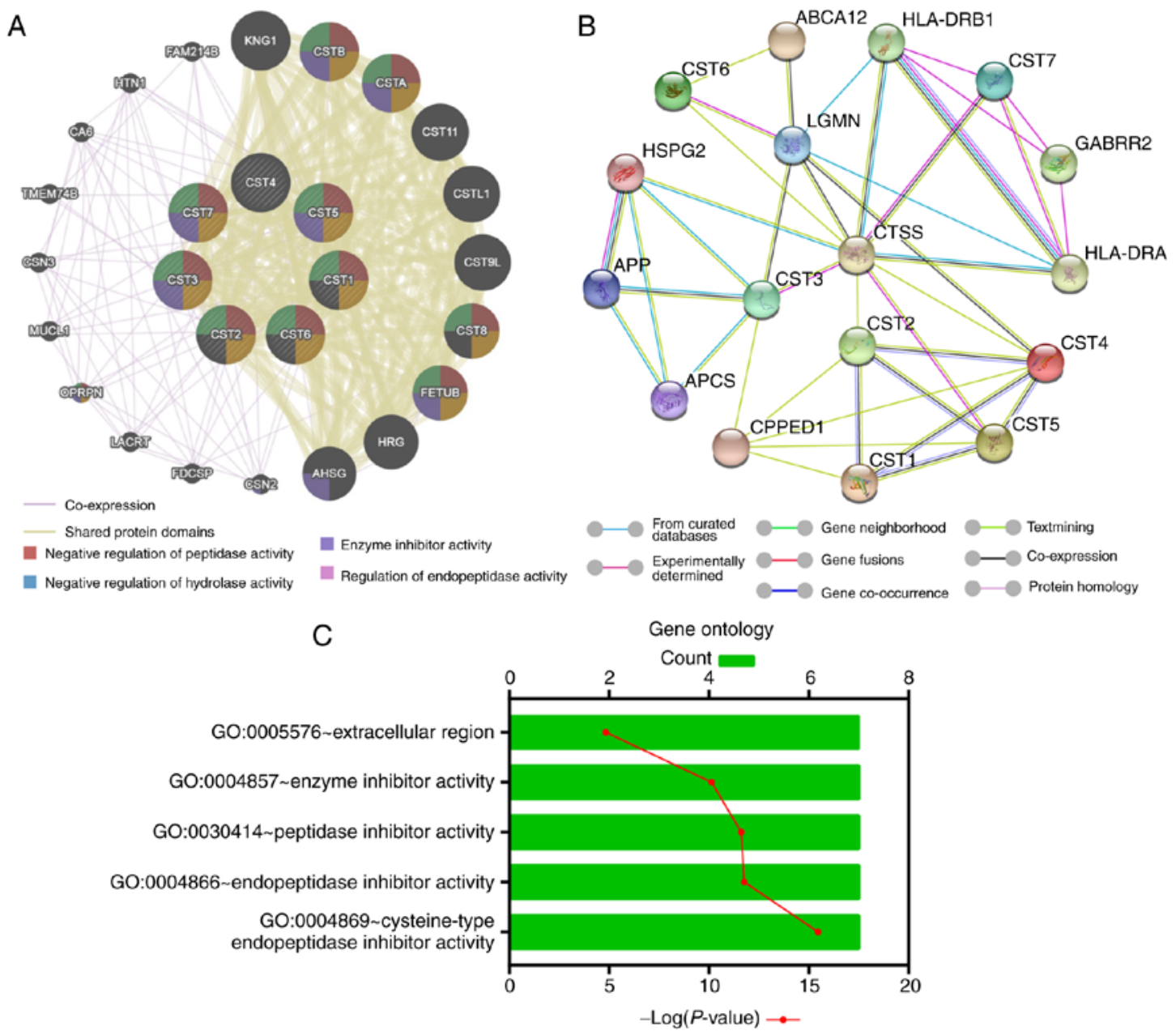

Figure 1. Gene-gene, protein-protein interaction networks and Gene Ontology term analysis of the cystatin gene family. (A) Gene-to-gene interaction network generated by GeneMANIA. (B) Protein-to-protein interaction network analyzed using STRING. (C) Gene Ontology term analysis in DAVID. STRING, Search Tool for the Retrieval of Interacting Genes/Proteins; DAVID, Database for Annotation, Visualization and Integrated Discovery.

Expression of the CST gene family in tissues. The results of MERAV revealed that the expression of the cystatin gene family was different between pancreas tumor and paraneoplastic tissues (Fig. 2A). Expression of CST1, CST2, CST4 and CST6 was upregulated in pancreas tumors. The median CST7 mRNA level was increased in tumor tissues but the difference was not statistically significant. From another perspective, CST3 and CST5 mRNA levels were downregulated in pancreas tumor tissues. Moreover, in the GTEx analysis of various normal tissues, CST3 expression was higher than that of other cystatin isoforms (Fig. 2B). CST7 is partly upregulated in whole blood and spleen. Co-expression relationships between CST genes in tumor tissues, as evaluated using Pearson's correlation, revealed that CST4 and CST5 had a positive correlation (Fig. S1).

Survival analysis of the cystatin gene family in TCGA early-stage PDAC patients. The relationship between clinicopathological features and prognosis of patients with early-stage PDAC in TCGA is presented in Table SI. All patients were divided into groups according to median values or stages of the clinicopathological features. In the univariate survival analysis, high CST7 expression was related to low-risk in the OS and DFS of PDAC (Fig. 3A and B and Table I). Based on the regression coefficient $(\beta=-0.709)$ in univariate Cox proportional hazards regression, prognosis risk had a negative correlation with CST7 expression level (Fig. 3C and Table I). Patients diagnosed with PDAC had longer OS and DFS when they harbored higher CST7 expression (Fig. 3D). Adjusted pathologic stage $\mathrm{T}$, pathologic stage $\mathrm{N}$, histologic grade, radical resection and targeted molecular therapy in the multivariate survival analysis, CST2, CST3 and CST7 were associated with the OS of PDAC patients. Higher CST2, CST3, and CS7 expression level have a lower risk in OS of PDAC patients ( $\mathrm{HR}=0.46,0.50$ and 0.44 , respectively; Table I). However, none of the cystatin gene family members were associated with DFS in early-stage PDAC. In this procedure, CST7 was selected as a key cystatin isoform.

To investigate the correlation between CTS7 expression and clinical features in the prognosis of PDAC patients, we performed a combined analysis of CST7 mRNA level with age, sex, tumor dimension and residual tumor status. The results revealed that high CST7 expression in groups with age $\leq 60$ years, females, tumor dimension and radical resection had a favorable outcome in PDAC patients (all $\mathrm{P}<0.05$; Fig. 4). Adjusting for number of positive lymph nodes, histologic grade, radiation therapy and targeted molecular therapy in multivariate regression analysis, groups of high CST7 expression 

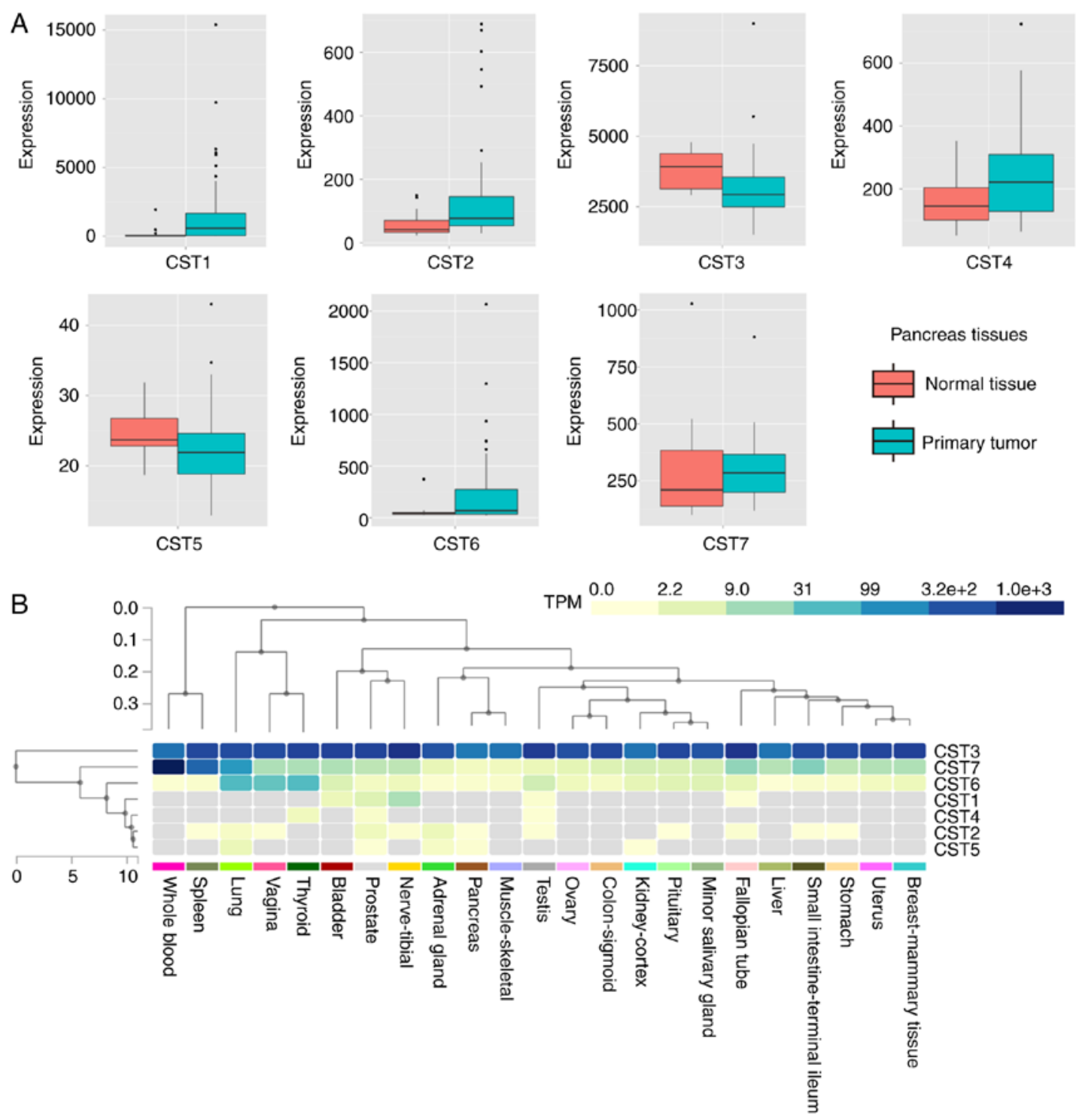

Figure 2. Cystatin gene family expression in (A) TCGA pancreas tissues, and (B) heatmap of expression in GTEx normal tissues. TCGA, The Cancer Genome Atlas; GTEx, Genotype-Tissue Expression.

and age $\leq 60$ years, male/female, dimension $>3 \mathrm{~cm}$ and radical resection had a lower risk in the OS of PDAC patients (all $\mathrm{P}<0.05$; Table II). In the DFS of PDAC patients, HRs for high CST7 expression and age $>60$ years, male/female, dimension $\leq 3 \mathrm{~cm}$ and radical resection were $0.14,0.17 / 0.24,0.23$ and 0.17 , respectively (Table II).

Prognosis model for CST7 expression in early-stage PDAC. To predict the clinical outcome risk in different CST7 mRNA levels and clinicopathological features, we constructed a prognosis nomogram for OS and DFS of PDAC patients $(\mathrm{C}$-index $=0.799$ and 0.772 , respectively). CST7 expression, histological grade, lymph node metastasis, resection status, therapy methods were associated with the OS of PDAC patients (Fig. 5A). Moreover, histological grade played an important role in the recurrence of PDAC and had a synergism with CST7 expression, lymph node metastasis, resection status, and tumor stages (Fig. 5B).

GSEA for CST7 in PDAC. In the GSEA, we identified that CST7 may be involved in immunomodulation, immune response, and cellular immune regulation (Fig. 6A-I). In terms of the biological process of PDAC patients, CST7 was associated with cell adhesion molecule CAMS and played a role in cell adhesion (Fig. 6J-L).

\section{Discussion}

In the present study, we analyzed the biological functions of family 2 cystatins using bioinformatics analysis. Gene expression levels of family 2 cystatins differed between paraneoplastic and pancreas tumor tissues. Furthermore, we identified CST7 as a key cystatin subunit in patients with early-stage PDAC in TCGA. DFS and OS of patients with surgically resected PDACs were significantly prolonged in the high-CST7 expression subgroup compared with the low-CST7 expression subgroup. Moreover, we investigated the prognostic value of CST7 using combined analysis and constructed a prognostic model for PDAC patients after pancreatectomy. The result of the GSEA demonstrated that CST7 may be involved in immune regulation. 

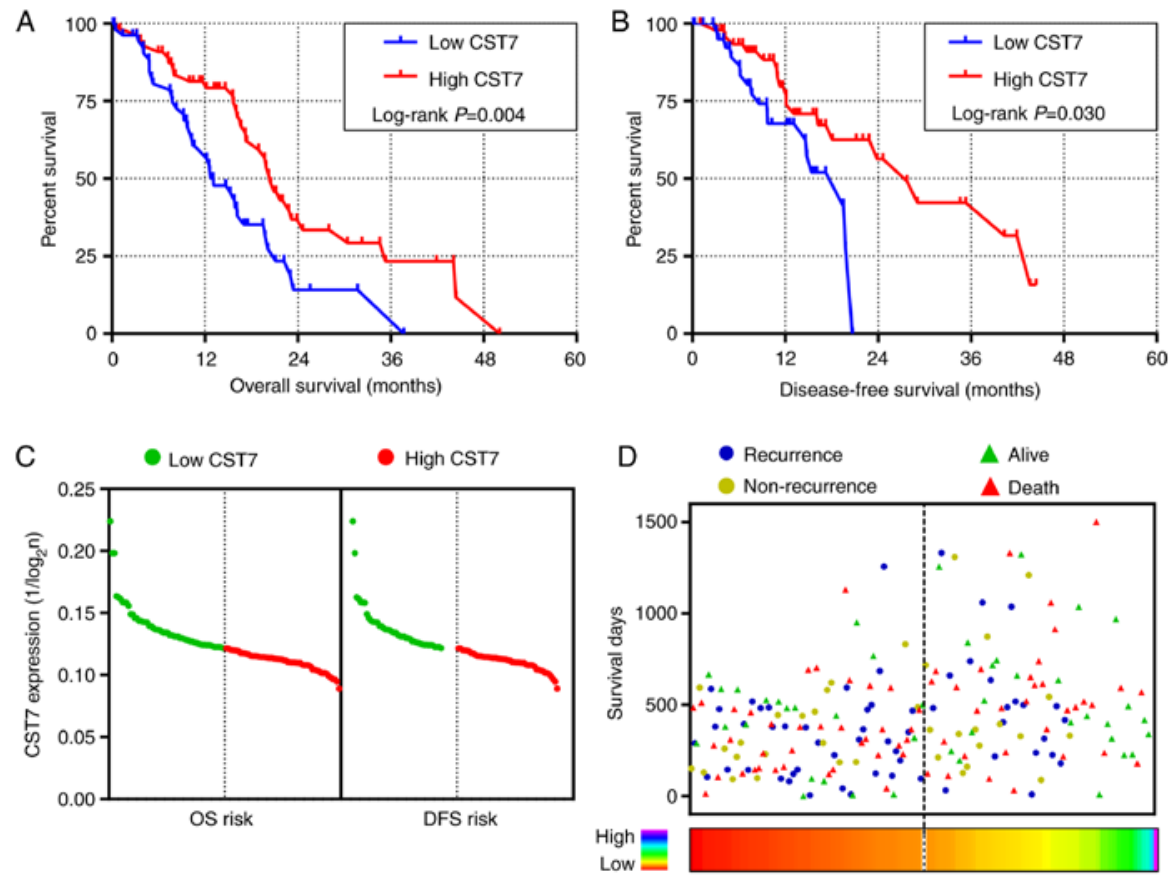

Figure 3. Survival analysis and risk evaluation of different CST7 expression levels in patients with early-stage PDAC. (A and B) Kaplan-Meier survival curves of OS and DFS for high- and low-CST7 expression groups. (C) OS and DFS risk in different CST7 expression levels. (D) Survival status distribution of PDAC patients and heatmap of CST7 gene expression for low- and high-risk groups. PDAC, pancreatic ductal adenocarcinoma; OS, overall survival; DFS, disease-free survival; CST7, cystatin F.
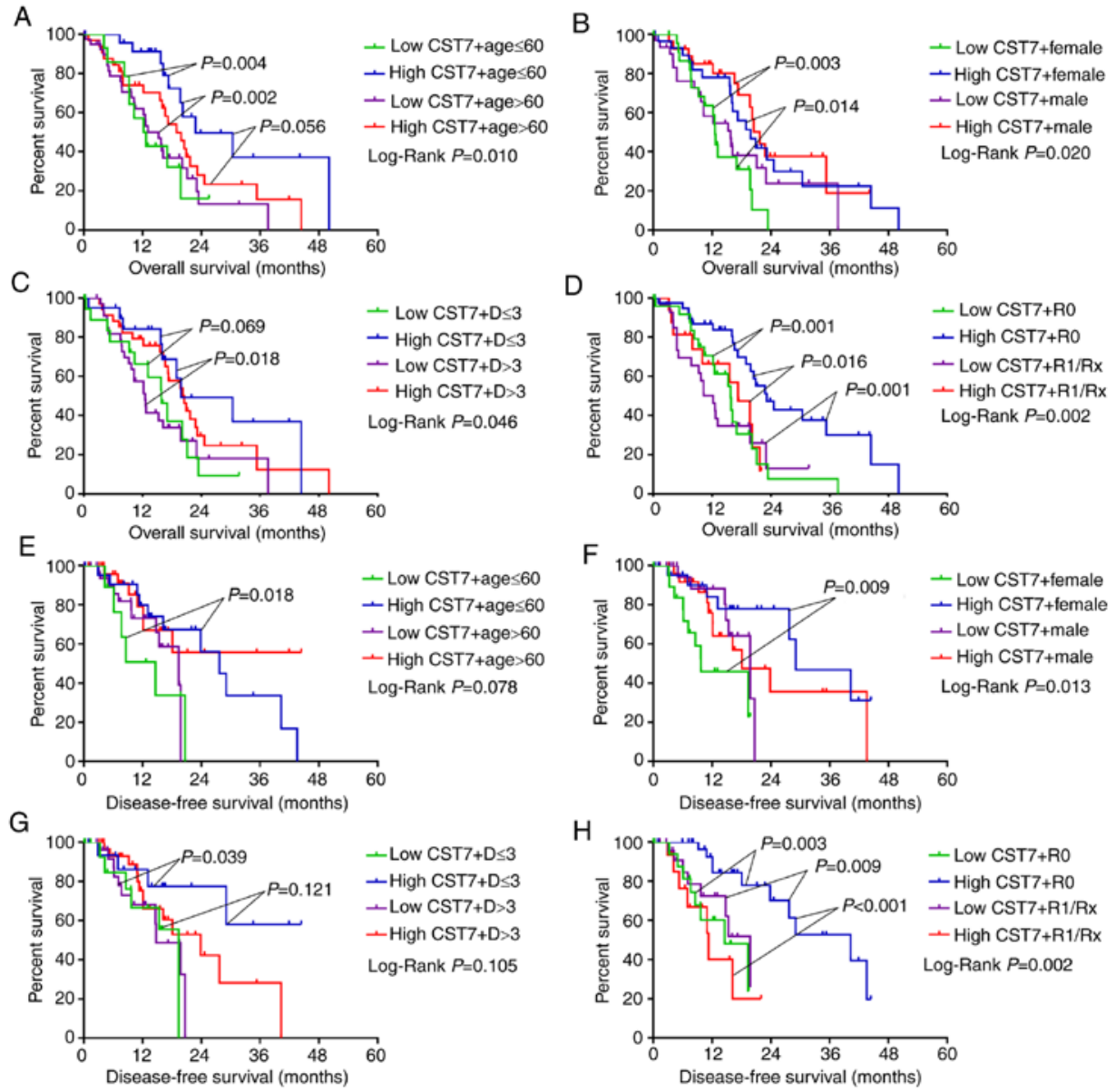

Figure 4. Combined survival analysis of CST7 expression and clinicopathological features in patients with early-stage PDAC. (A-D) Kaplan-Meier survival curves of OS for high and low CST7 expression combined with age, sex, tumor dimension, and residual tumor status, respectively. (E-H) Kaplan-Meier survival curves of DFS for high and low CST7 expression combined with age, sex, tumor dimension, and residual tumor status, respectively. CST7, cystatin F; PDAC, pancreatic ductal adenocarcinoma; OS, overall survival; DFS, disease-free survival. 


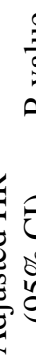

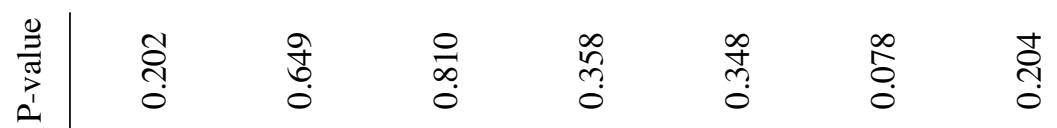

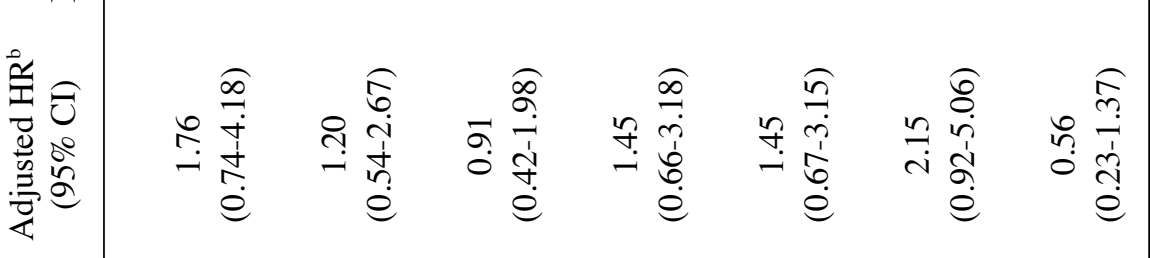

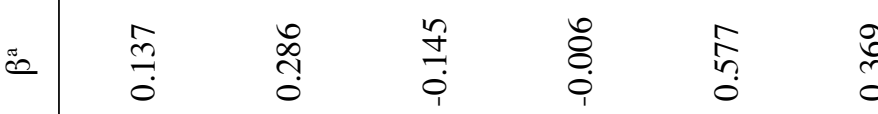

0
0
0

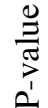

:े

\begin{tabular}{lll} 
Iี & \multirow{2}{*}{$:$} & 0 \\
0 & 0 & 0 \\
0 & 0
\end{tabular}

\&

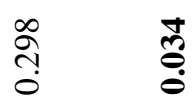

葛

@े

$\stackrel{\leftrightarrow}{8}$

올

तิ क्षे

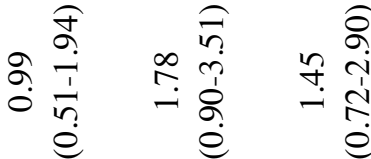

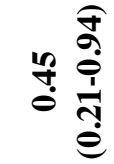

党

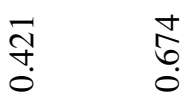

苔

\&̊ำ

ô.

กิ

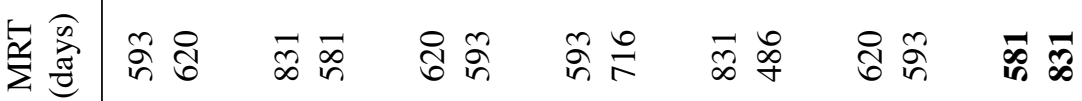

苞

:

$\stackrel{\infty}{0}$

$\stackrel{n}{3} \quad \stackrel{0}{0}$

$\stackrel{\substack{0 \\ 0}}{\stackrel{n}{n}}$

$\underset{n}{n}$

$\stackrel{\square}{\stackrel{\Delta}{8}}$

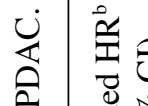

密

卧

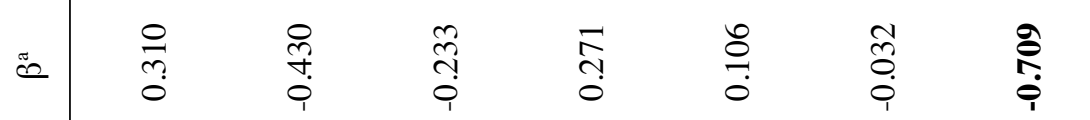

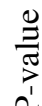

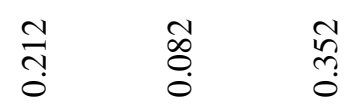

तิ

$\stackrel{\substack{0 \\ \infty}}{0}$

$\stackrel{10}{8}$

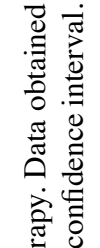

졸

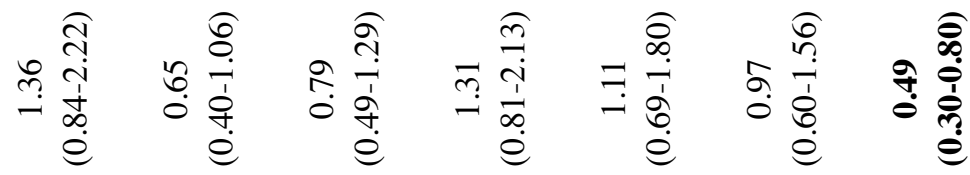

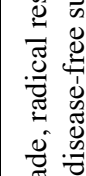

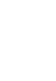

㱐

要

.

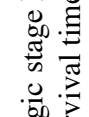

है छ

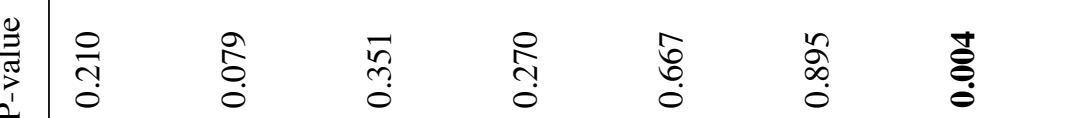

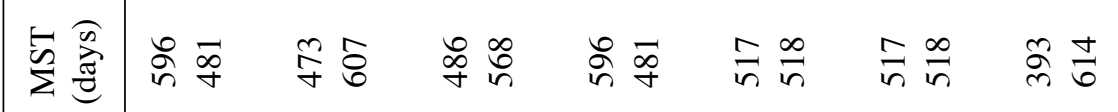

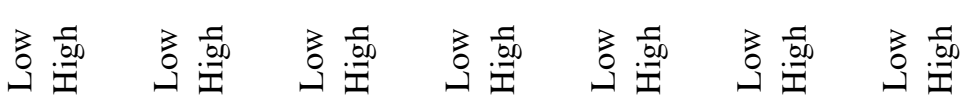

惫

5

产

ह

喜

窎

总

E 


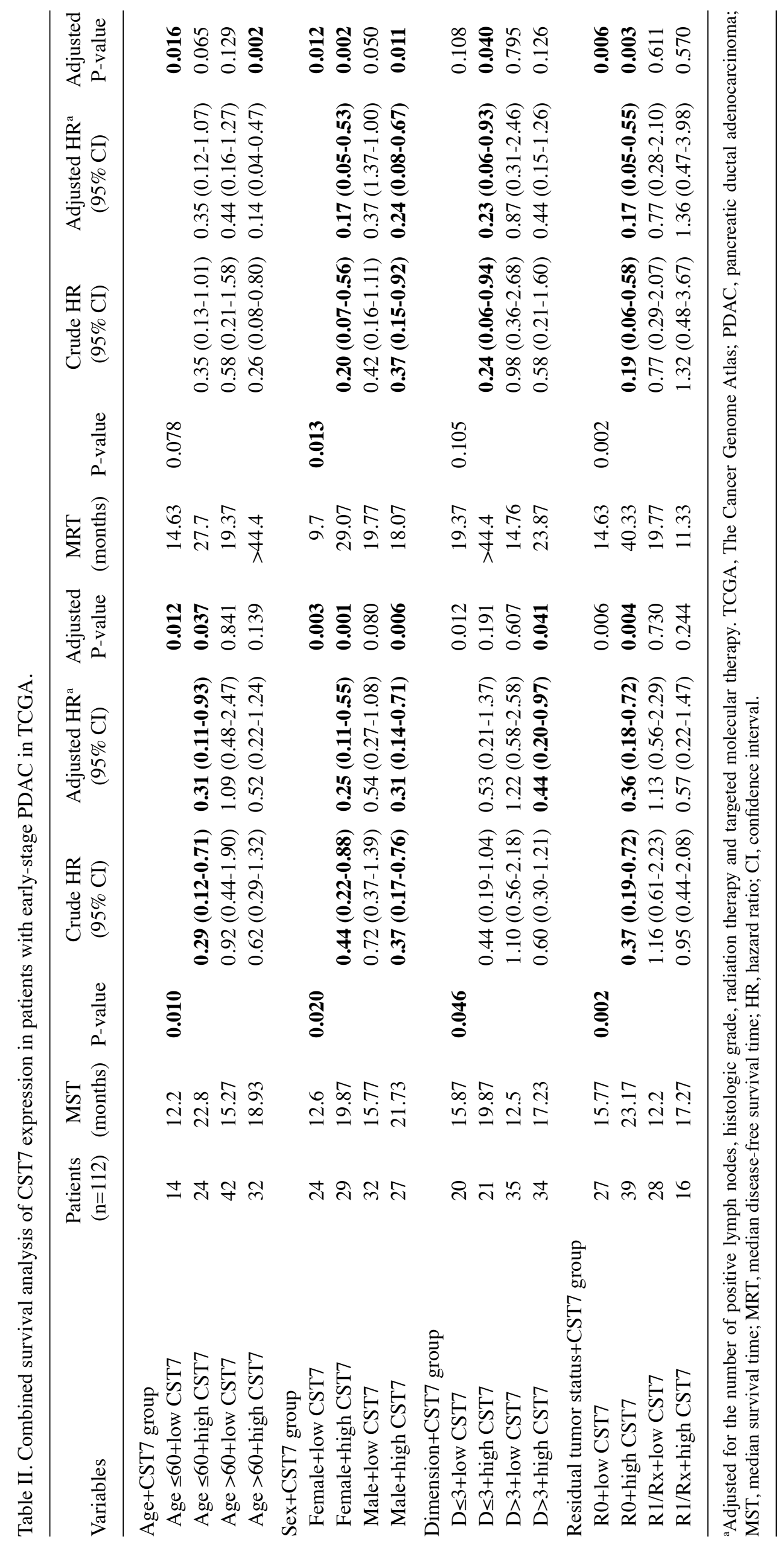


A

Points
Age
Sex
Number_of_lymphnodes_
positive_by_HE
Pathologic_T
Neoplasm_histologic_grade
Residual_tumor
Radiation_therapy
Targeted_molecular_therapy
CST7_mRNA
Total points
1-year overall survival
2-years overall survival
3-years overall survival

B

Points
Age
Sex
Maximum_tumor_dimension
number_of_lymphnodes_
positive_by_HE
pathologic_T
Neoplasm_histologic_grade
Residual_tumor
Radiation_therapy
Targeted_molecular_therapy
CST7_mRNA
Total points
1-year recurrence-free survival
2-years recurrence-free survival
3-years recurrence-free survival

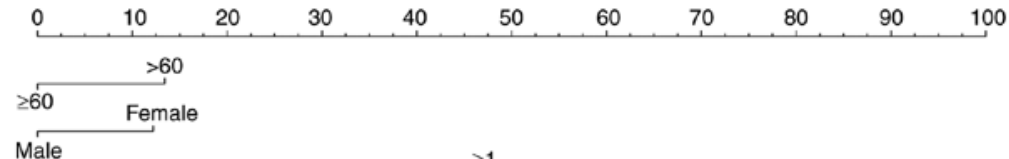

$0_{\mathrm{T} 1+\mathrm{T} 2}$

T3 G1

G2 R1/Rx G3+G4

RO No

Yes No

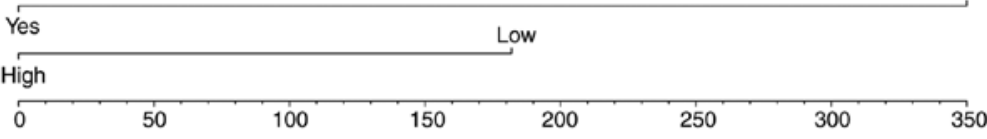

\begin{tabular}{|c|c|c|c|c|c|c|}
\hline 0 & 50 & 100 & 150 & 200 & 250 & 300 \\
\hline
\end{tabular}

$\begin{array}{lllllllll}0.9 & 0.8 & 0.7 & 0.6 & 0.5 & 0.4 & 0.3 & 0.2 & 0.1\end{array}$

$\begin{array}{lllllllll}0.8 & 0.7 & 0.6 & 0.5 & 0.4 & 0.3 & 0.2 & 0.1\end{array}$

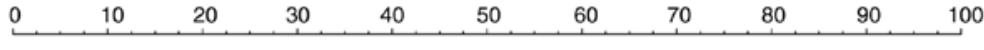

$\geq 60$

\begin{tabular}{|c|c|c|}
\hline$>60$ & Female & \\
\hline Male & $>3$ & \\
\hline$\geq 3$ & & $\geq 1$ \\
\hline 0 & & T3 \\
\hline $1+\mathrm{T} 2$ & & G2 \\
\hline G1 & & $\mathrm{R} 1 / \mathrm{Rx}$ \\
\hline
\end{tabular}

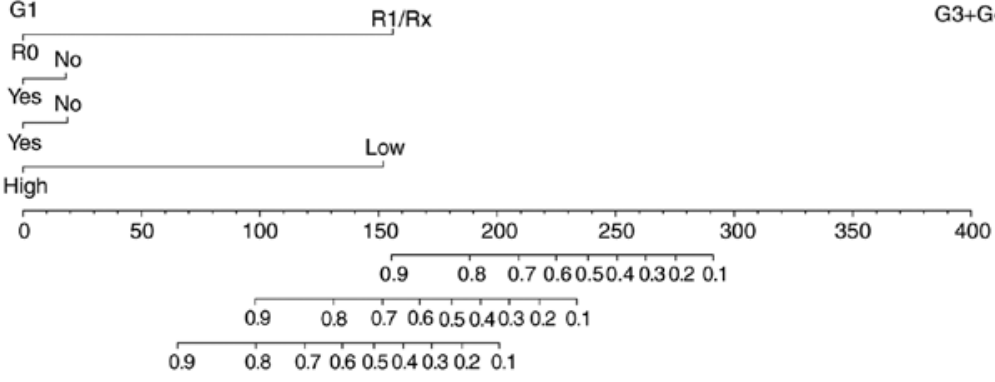

Figure 5. Nomogram model analysis of CST7 expression level for (A) OS and (B) DFS in patients with early-stage PDAC. CST7, cystatin F; OS, overall survival; DFS, disease-free survival; PDAC, pancreatic ductal adenocarcinoma.

As the most commonly used tumor biomarker for detecting PDAC, the main limitations of CA 19-9 include its frequent elevations associated with non-malignant diseases such as pancreatitis and obstructive jaundice, and the inability to detect many early-stage tumors (23). CA 19-9 is also not suitable for estimating $5-10 \%$ of patients who are carriers of the Lewis-negative genotype and advance malignancies that do not express antigen (24). Thus, it is extremely important and necessary to screen additional key genes associated with PDAC that may act as diagnostic, prognostic or therapeutic biomarkers for improving the diagnostic ability of CA19-9.

After screening, CST7 was considered to be a key cystatin subunit in early-stage PDAC. Using GSEA, we identified that CST7 was related to the regulation of immune cell activity. Previous studies demonstrated that CST7 expression may play roles in regulating the activation of natural killer cells, differentiation of monocytes to macrophages and influenza vaccine responses (25-27). CST7 encodes a glycosylated cysteine protease inhibitor with a putative role in immune regulation through inhibition of a unique target in the hematopoietic system. It has been reported that CST7 is mainly expressed by immune cells $(28,29)$ where it is often elevated when these cells differentiate or activate from a stationary precursor. Cystatins are considered to be typical emergency inhibitors, trapping proteases that escape from endosomes/lysosomes or cells, and converting them into proteolytically inactive complexes (30). However, the amino acids present in the $\mathrm{N}$-terminal region of the protease-binding loop and CST7 differ from the amino acids of other family members, indicating that CST7 may bind to different protease targets. Compared with other cystatins, core sugars of the N-linked glycosylation sites of CST7 are highly structured, and conformation and interactions with the combined proteins indicate that unique features of CST7 may modulate its inhibitory properties though structural reconstruction (31).

In the present study, GO term annotations revealed that the cystatin family plays roles in the extracellular region and demonstrates inhibitory activity against biologically active proteins. A recent study demonstrated that CST7 regulated intracellular, but not extracellular, protease activity by targeting cathepsin C (32). CST7 plays an important role in activation of various serine protease zymogens in secretory granules of tumor-related immune cells, such as cytotoxic T lymphocytes, mast cells, NK cells, and neutrophils (33-35). 
A

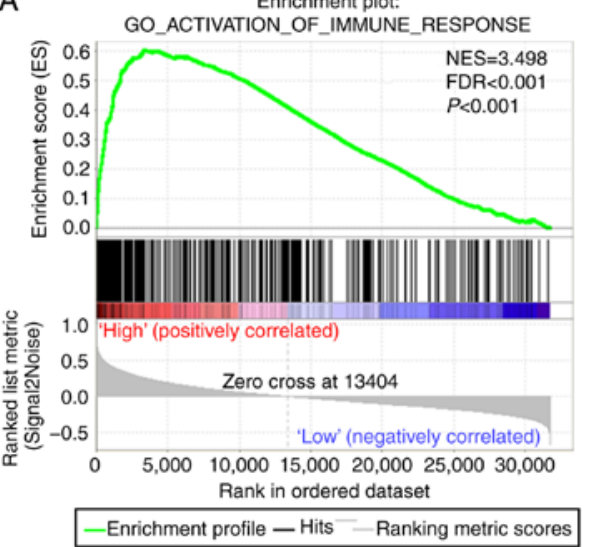

C Enrichment plot:GO_ADAPTIVE_IMMUNE_RESPONSE

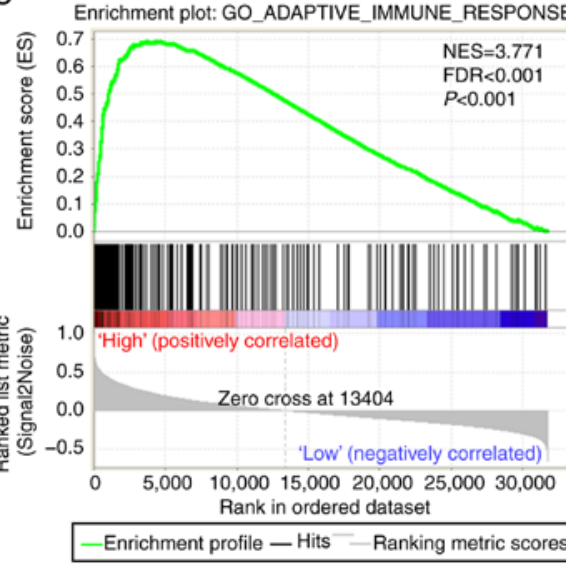

E
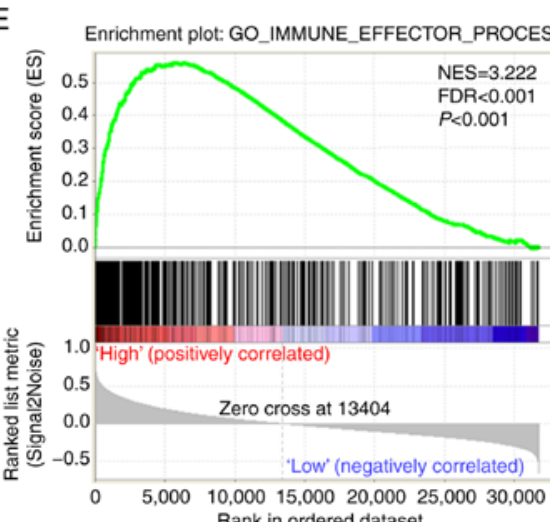

-Enrichment profile - Hits - - Ranking metric scores

G

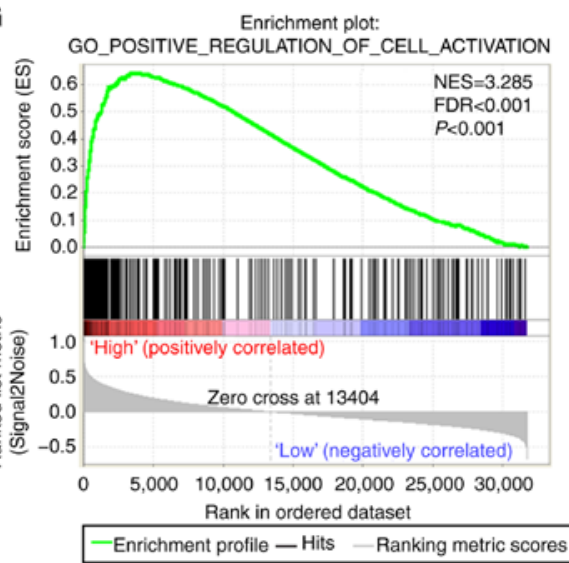

B

B Enrichment plot:

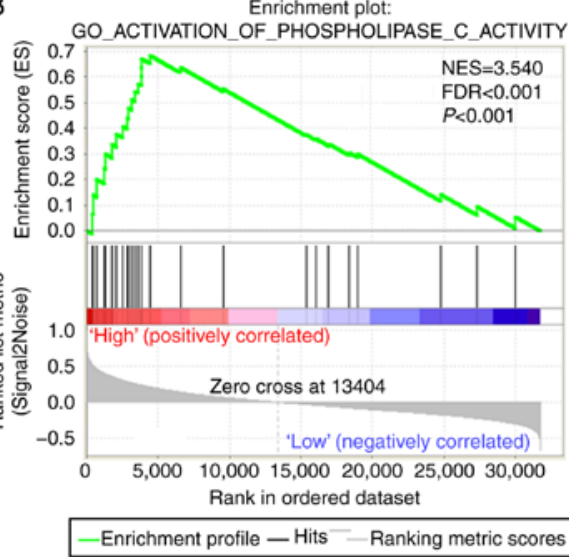

D Enrichment plot:

GO_ANTIGEN_RECEPTOR_MEDIATED_SIGNALING_
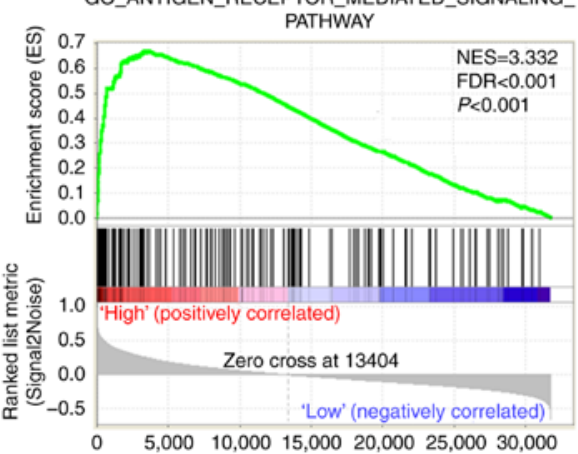
Rank in ordered dataset

-Enrichment profile - Hits $^{-}-$Ranking metric scores
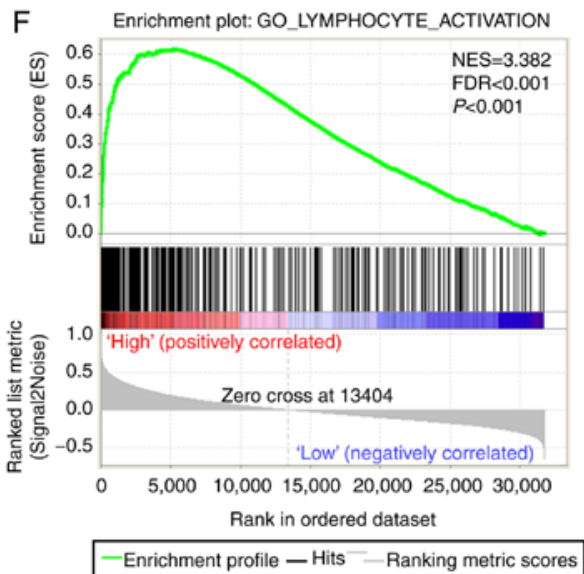

$\mathrm{H} \quad$ Enrichment plot:

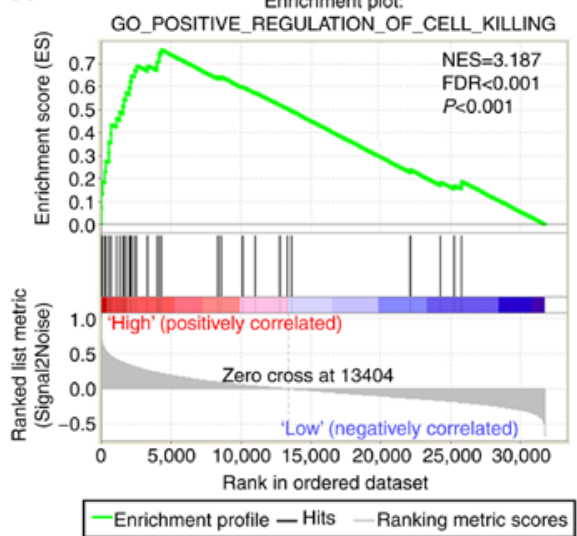

Figure 6. GSEA of CST7 expression in patients with early-stage PDAC. (A-J) GSEA report for high CST7 expression using high- vs. low-risk group. 
I
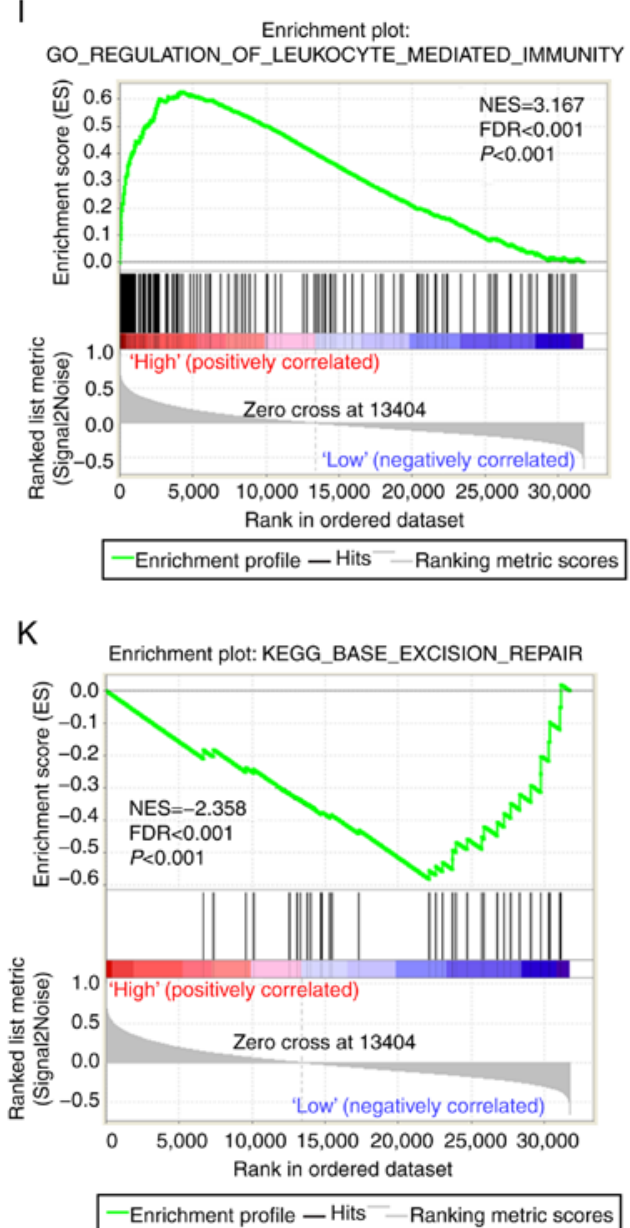

$J$ GO Enrichment plot:

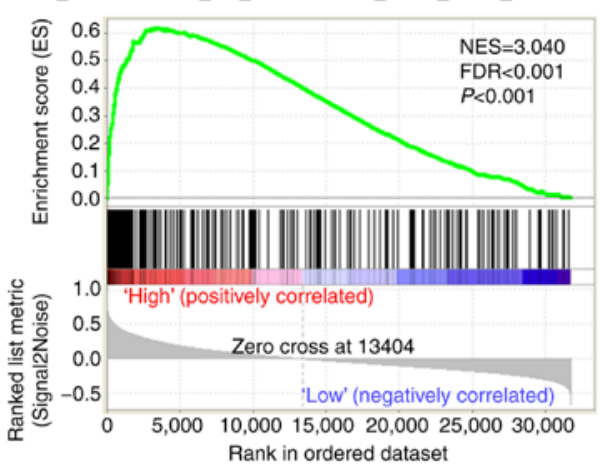

Enrichment profile - Hits-- Ranking metric scores

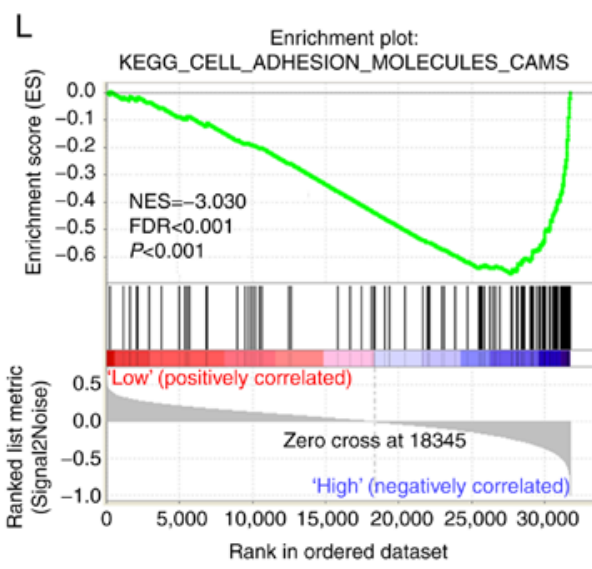

Enrichment profile - Hits - - Ranking metric scores

Figure 6. Continued. GSEA of CST7 expression in patients with early-stage PDAC. (I-J) GSEA report for high CST7 expression using high- vs. low-risk group. (K) GSEA report for low CST7 expression using high- vs. low-risk group. (L) GSEA report for high CST7 expression using low- vs. high-risk group. GSEA, gene set enrichment analysis; CST7, cystatin F; PDAC, pancreatic ductal adenocarcinoma.

Although destruction of the CST7 dimer is enough to produce an endopeptidase inhibitor such as cathepsin $\mathrm{L}$, a proteolytic cleavage event is applied for converting inactive CST7 into an active cathepsin $\mathrm{C}$ inhibitor. Cathepsin $\mathrm{C}$ is essential for the activation of cytokines, such as TNF- $\alpha$ and IL- $1 \beta$. CST7 was revealed specifically to bind and inhibit cathepsin $\mathrm{C}$ in immune cells via regulation of split anergy $(32,36)$.

Moreover, expression of CST7 has been observed in various human cancer cell lines established from malignant tumors. As the only family 2 cystatin able to enter endosomal/lysosomal vesicles and to regulate directly the activity of intracellular cysteine cathepsins, CST7 is highly upregulated in promonocytic U937 and promyeloblast HL-60 cells (26). Pierre and Mellman (37) reported that the apparent distribution of smaller vesicles/particles in U937 cells indicates that CST7 is partially localized to organelles that resemble fusion lysosomes/endosomes, wherein antigen and constant chain processing occurs in antigen-presenting cells. Immunocytochemical staining of CST7 in human promonocyte U937 cells displays a vesicular pattern (38).

Furthermore, recent studies have revealed that CST7 expression level was associated with tumor progression and served as an independent factor for liver metastasis in colorectal cancer patients $(39,40)$. The prognosis of the patients with a higher expression of CST7 was significantly worse than those with a lower expression. However, our results indicated that high CST7 expression level may be a protective factor for patients with early-stage PDAC. Using the Human Protein Atlas project (HPA; https://www.proteinatlas.org/), an online visualization website for detecting the relationship between the protein level and clinical outcomes based on TCGA data, we determined that the protein level of CST7 was not associated with the prognosis of PDAC patients but would be a potential prognostic marker in renal cancer, endometrial, head and neck, breast and cervical cancer. We speculate that this gene has tissue differences in tumor prognosis and prognosis evaluation. CST7 may serve as a tumor suppressor gene in PDAC patients, and high CST7 expression promotes autoimmune cell monitoring. For our prognostic model constructed using a nomogram, CST7 harbored a high predictive effect on the clinical outcomes of PDAC and could assist clinicians to judge disease outcomes and treatment. Considering the lack of independent data and some missing clinical data which are limitations of the present study, an additional large verification cohort to validate the results is necessary.

In conclusion, the results of the present study indicated that CST7 could be a useful biomarker for the prognostic prediction of early-stage PDAC. 


\section{Acknowledgements}

We are grateful to TCGA group for providing relevant data.

\section{Funding}

The present study was supported in part by the National Natural Science Foundation of China (nos. 81560535, 81802874, 81072321, 30760243, 30460143 and 30560133), Natural Science Foundation of Guangxi Province of China (grant no. 2018GXNSFBA138013 and 2018GXNSFAA050119), 2009 Program for New Century Excellent Talents in University (NCET), Guangxi Natural Sciences Foundation (no. GuiKeGong 1104003A-7), and Guangxi Health Ministry Medicine Grant (Key-Scientific Research-Grant Z201018). This study was also partly supported by the Scientific Research Fund of the Health and Family Planning Commission of Guangxi Zhuang Autonomous Region (Z2016318), Key Laboratory of High-Incidence-Tumor Prevention and Treatment (Guangxi Medical University), Ministry of Education (GKE2018-01), the Guangxi Key R\&D Program (GKEAB18221019), The Basic Ability Improvement Project for Middle-aged and Young Teachers in Colleges and Universities in Guangxi (2018KY0110), Innovation Project of Guangxi Graduate Education (JGY2018037), and 2018 Innovation Project of Guangxi Graduate Education (YCBZ2018036). Furthermore, the present study was also partly supported by the Research Institute of Innovative Think-tank in Guangxi Medical University (The gene-environment interaction in hepatocarcinogenesis in Guangxi HCCs and its translational applications in the HCC prevention). We would also acknowledge the support of the National Key Clinical Specialty Programs (General Surgery and Oncology) and the Key Laboratory of Early Prevention and Treatment for Regional High-IncidenceTumor (Guangxi Medical University), Ministry of Education, China.

\section{Availability of data and materials}

All data generated or analyzed during the present study are included in this published article.

\section{Authors' contributions}

CY and TP designed the study; CY, CH, XY and TY performed research; XY, XW, XL, ZL and TY provided sample collection and clinical support; GZ, TY and WQ contributed to data interpretation. CY and ZL wrote the manuscript, and XL and TP critically revised the manuscript and participated in the analysis and interpretation of the data. All authors reviewed, edited and approved the final version of the manuscript and agree to be accountable for all aspects of the research in ensuring that the accuracy or integrity of any part of the work are appropriately investigated and resolved.

\section{Ethics approval and consent to participate}

The present study was approved by the Ethics Committee of The First Affiliated Hospital of Guangxi Medical University (Guangxi, China).

\section{Patient consent for publication}

Not applicable.

\section{Competing interests}

The authors declare that they have no competing interests.

\section{References}

1. Li D, Xie K, Wolff R and Abbruzzese JL: Pancreatic cancer. Lancet 363: 1049-1057, 2004.

2. Siegel R, Naishadham D and Jemal A: Cancer statistics, 2012. CA Cancer J Clin 62: 10-29, 2012.

3. Jemal A, Siegel R, Ward E, Murray T, Xu J and Thun MJ: Cancer statistics, 2007. CA Cancer J Clin 57: 43-66, 2007.

4. Siegel RL, Miller KD and Jemal A: Cancer statistics, 2017. CA Cancer J Clin 67: 7-30, 2017.

5. Conrad C and Lillemoe KD: Surgical palliation of pancreatic cancer. Cancer J 18: 577-583, 2012.

6. Vincent A, Herman J, Schulick R, Hruban RH and Goggins M: Pancreatic cancer. Lancet 378: 607-620, 2011.

7. Choi EH, Kim JT, Kim JH, Kim SY, Song EY, Kim JW, Kim SY, Yeom YI, Kim IH and Lee HG: Upregulation of the cysteine protease inhibitor, cystatin SN, contributes to cell proliferation and cathepsin inhibition in gastric cancer. Clin Chim Acta 406: 45-51, 2009.

8. Travis $\mathbf{J}$ and Potempa $\mathbf{J}$ : Bacterial proteinases as targets for the development of second-generation antibiotics. Biochim Biophys Acta 1477: 35-50, 2000.

9. Koblinski JE, Ahram M and Sloane BF: Unraveling the role of proteases in cancer. Clin Chim Acta 291: 113-135, 2000.

10. Kos J and Lah TT: Cysteine proteinases and their endogenous inhibitors: Target proteins for prognosis, diagnosis and therapy in cancer (review). Oncol Rep 5: 1349-1410, 1998.

11. Verbovšek U, Van Noorden CJ and Lah TT: Complexity of cancer protease biology: Cathepsin K expression and function in cancer progression. Semin Cancer Biol 35: 71-84, 2015.

12. Cox J: Cystatins as regulators of cancer. Med Res Arch 5: 2017.

13. Barrett AJ: The cystatins: A new class of peptidase inhibitors. Trends Biochem Sci 12: 193-196, 1987.

14. Henskens YM, Veerman EC and Nieuw Amerongen AV: Cystatins in health and disease. Biol Chem Hoppe Seyler 377: 71-86, 1996.

15. Abrahamson M, Alvarez-Fernandez $M$ and Nathanson $C M$ : Cystatins. Biochem Soc Symp 179-199, 2003.

16. Chimonidou M, Tzitzira A, Strati A, Sotiropoulou G, Sfikas C, Malamos N, Georgoulias V and Lianidou E: CST6 promoter methylation in circulating cell-free DNA of breast cancer patients. Clin Biochem 46: 235-240, 2013.

17. Cao X, Li Y, Luo RZ, Zhang L, Zhang SL, Zeng J, Han YJ and Wen ZS: Expression of Cystatin SN significantly correlates with recurrence, metastasis, and survival duration in surgically resected non-small cell lung cancer patients. Sci Rep 5: 8230, 2015.

18. Alvarez-Díaz S, Valle N, García JM, Peña C, Freije JM, Quesada V, Astudillo A, Bonilla F, López-Otín C and Muñoz A: Cystatin D is a candidate tumor suppressor gene induced by vitamin $\mathrm{D}$ in human colon cancer cells. J Clin Invest 119: 2343-2358, 2009.

19. Hünten S and Hermeking H: p53 directly activates cystatin D/CST5 to mediate mesenchymal-epithelial transition: A possible link to tumor suppression by vitamin D3. Oncotarget 6: 15842-15856, 2015.

20. Liao X, Huang K, Huang R, Liu X, Han C, Yu L, Yu T, Yang C, Wang $X$ and Peng T: Genome-scale analysis to identify prognostic markers in patients with early-stage pancreatic ductal adenocarcinoma after pancreaticoduodenectomy. Onco Targets Ther 10: 4493-4506, 2017.

21. Harrell FE Jr: rms: Regression modeling strategies. R package version 4.0-0. City. 2013.

22. Harrell FE Jr, Califf RM, Pryor DB, Lee KL and Rosati RA: Evaluating the yield of medical tests. JAMA 247: 2543-2546, 1982.

23. Koopmann J, Rosenzweig CN, Zhang Z, Canto MI, Brown DA, Hunter M, Yeo C, Chan DW, Breit SN and Goggins M: Serum markers in patients with resectable pancreatic adenocarcinoma: Macrophage inhibitory cytokine 1 versus CA19-9. Clin Cancer Res 12: 442-446, 2006. 
24. Locker GY, Hamilton S, Harris J, Jessup JM, Kemeny N, Macdonald JS, Somerfield MR, Hayes DF and Bast RC Jr; ASCO: ASCO 2006 update of recommendations for the use of tumor markers in gastrointestinal cancer. J Clin Oncol 24: 5313-5327, 2006.

25. Maher K, Konjar S, Watts C, Turk B and Kopitar-Jerala N Cystatin $\mathrm{F}$ regulates proteinase activity in IL-2-activated natural killer cells. Protein Pept Lett 21: 957-965, 2014.

26. Dautović E, Perišić Nanut M, Softić A and Kos J: The transcription factor $\mathrm{C} / \mathrm{EBP} \alpha$ controls the role of cystatin $\mathrm{F}$ during the differentiation of monocytes to macrophages. Eur J Cell Biol 97: 463-473, 2018.

27. Voigt EA, Grill DE, Zimmermann MT, Simon WL, Ovsyannikova IG, Kennedy RB and Poland GA: Transcriptomic signatures of cellular and humoral immune responses in older adults after seasonal influenza vaccination identified by data-driven clustering. Sci Rep 8: 739, 2018.

28. Halfon S, Ford J, Foster J, Dowling L, Lucian L, Sterling M, Xu Y, Weiss M, Ikeda M, Liggett D, et al: Leukocystatin, a new class II cystatin expressed selectively by hematopoietic cells. J Biol Chem 273: 16400-16408, 1998.

29. Ni J, Fernandez MA, Danielsson L, Chillakuru RA, Zhang J, Grubb A, Su J, Gentz R and Abrahamson M: Cystatin F is a glycosylated human low molecular weight cysteine proteinase inhibitor. J Biol Chem 273: 24797-24804, 1998.

30. Turk B, Turk D and Salvesen GS: Regulating cysteine protease activity: Essential role of protease inhibitors as guardians and regulators. Curr Pharm Des 8: 1623-1637, 2002.

31. Schüttelkopf AW, Hamilton G, Watts C and van Aalten DM: Structural basis of reduction-dependent activation of human cystatin F. J Biol Chem 281: 16570-16575, 2006.

32. Hamilton G, Colbert JD, Schuettelkopf AW and Watts C: Cystatin F is a cathepsin C-directed protease inhibitor regulated by proteolysis. EMBO J 27: 499-508, 2008.
33. Pham CT and Ley TJ: Dipeptidyl peptidase I is required for the processing and activation of granzymes A and B in vivo. Proc Natl Acad Sci USA 96: 8627-8632, 1999.

34. Wolters PJ, Pham CT, Muilenburg DJ, Ley TJ and Caughey GH Dipeptidyl peptidase I is essential for activation of mast cell chymases, but not tryptases, in mice. J Biol Chem 276: 18551-18556, 2001

35. Adkison AM, Raptis SZ, Kelley DG and Pham CT: Dipeptidyl peptidase I activates neutrophil-derived serine proteases and regulates the development of acute experimental arthritis. J Clin Invest 109: 363-371, 2002.

36. Magister $\dot{\mathrm{S}}$, Tseng HC, Bui VT, Kos J and Jewett A: Regulation of split anergy in natural killer cells by inhibition of cathepsins $\mathrm{C}$ and $\mathrm{H}$ and cystatin F. Oncotarget 6: 22310-22327, 2015.

37. Pierre P and Mellman I: Developmental regulation of invariant chain proteolysis controls MHC class II trafficking in mouse dendritic cells. Cell 93: 1135-1145, 1998.

38. Nathanson CM, Wassélius J, Wallin $\mathrm{H}$ and Abrahamson M: Regulated expression and intracellular localization of cystatin $\mathrm{F}$ in human U937 cells. FEBS J Biochem 269: 5502-5511, 2002.

39. Georgieva M, Krasteva M, Angelova E, Ralchev K, Dimitrov V, Bozhimirov S, Georgieva E and Berger MR: Analysis of the $\mathrm{K}$-ras/B-raf/Erk signal cascade, p53 and CMAP as markers for tumor progression in colorectal cancer patients. Oncol Rep 20: $3-11,2008$.

40. Utsunomiya T, Hara Y, Kataoka A, Morita M, Arakawa H, Mori $M$ and Nishimura S: Cystatin-like metastasis-associated protein mRNA expression in human colorectal cancer is associated with both liver metastasis and patient survival. Clin Cancer Res 8: 2591-2594, 2002.

This work is licensed under a Creative Commons Attribution-NonCommercial-NoDerivatives 4.0 International (CC BY-NC-ND 4.0) License. 